\title{
Bildungsbezogene Erwartungen an Tagesschulen: Förderangebote an offenen Tagesschulen in der Deutschschweiz.
}

\section{Lukas Frei, Marianne Schüpbach, Benjamin von Allmen und Wim Nieuwenboom}

Im aktuellen Bildungsdiskurs wird Tagesschulen gerade im Hinblick auf Bildungsgerechtigkeit und verbesserter individueller Förderung eine zentrale Rolle eingeräumt. Ob Deutschschweizer Tagesschulen die entsprechenden Lerngelegenheiten bieten, ist allerdings kaum bekannt. Dieser Beitrag diskutiert bildungsbezogene Erwartungen an Tagesschulen sowie gängige Qualitätsmodelle und untersucht anhand offener Tagesschulen aus der Deutschschweiz, welche Rolle pädagogische Motive bei der Gründung von Tagesschulen spielen, wie stark sich Schulleitung und Lehrpersonen an der konzeptionellen Ausarbeitung beteiligen, welche individualisierenden und differenzierenden Förderelemente im Tagesschulangebot verankert sind und ob Letztere von Schulentwicklungsmerkmalen beeinflusst werden.

\section{Einle itung}

In den letzten 15 Jahren wurde das Angebot an Tagesschulen - Schulen, welche neben dem regulären Unterricht zusätzliche Bildungs- und Betreuungsangebote während des ganzen Tages bieten - in der Deutschschweiz deutlich ausgebaut (Stern et al., 2013). Verbunden mit der zunehmenden Dichte an Tagesschulen und dem ebenfalls stark intensivierten Ausbau ganztägiger Bildungs- und Betreuungsangebote in Deutschland, kam es im deutschsprachigen Raum zu einer vertieften Auseinandersetzung mit der Qualität entsprechender Angebote und deren Funktionen innerhalb des Bildungssystems (Fischer et al., 2011; Schüpbach, 2010). Da Tagesschulen die Möglichkeit für erweiterte Lerngelegenheiten ausserhalb des Unterrichts bieten, beispielsweise durch stärker individualisierende und differenzierende Förderangebote, wecken sie Hoffnungen auf eine verbesserte individuelle Förderung und mehr Bildungsgerechtigkeit. Allerdings ist bisher kaum bekannt, ob entsprechende Lerngelegenheiten in Deutschschweizer Tagesschulen überhaupt angeboten werden und welche Entwicklungsprozesse hinter dem Ausbau von Tagesschulen stehen. Dieser Beitrag untersucht, welche Motive dem Ausbau offener Tagesschulen in der Deutsch- 
schweiz zugrunde liegen, wie stark Lehrpersonen und Schulleitungen an konzeptionellen Festlegungen zu den Tagesschulangeboten beteiligt sind und inwieweit Aufgabenhilfe und -betreuung sowie stärker differenzierende Förderangebote zur Verfügung stehen. Zudem wird untersucht, ob sich Entwicklungsmerkmale identifizieren lassen, welche den Ausbau solcher Förderangebote begünstigen.

\section{Erwartungen an Tagesschulen}

Die Schweizerische Konferenz der kantonalen Erziehungsdirektoren definiert Tagesschulen als "Schulen mit ganztägigen Betreuungsangeboten (inklusive Mittagsverpflegung) an mehreren Tagen pro Woche» (EDK, 2013, S. 349), was in etwa der Definition von Ganztagsschulen in Deutschland entspricht (Sekretariat der Ständigen Konferenz der Kultusminister der Länder in der Bundesrepublik Deutschland, 2011). Einige Tagesschulen werden als gebundene Tagesschulen geführt, wobei sämtliche Kinder neben den Unterrichtszeiten auch an bestimmten ausserunterrichtlichen Angeboten teilnehmen müssen. Häufiger trifft man aber auf offene Tagesschulen, d.h. Schulen, die den Kindern nebst dem Unterricht ein modulares Bildungs- und Betreuungsangebot anbieten, welches freiwillig genutzt werden kann. Diese ausserunterrichtlichen Angebote - im Folgenden als Tagesschulangebote bezeichnet - stehen in der Regel unter Aufsicht und Verantwortung der Schulleitung und werden von einer Tagesschulangebotsleitung geführt.

Gesellschaftliche Veränderungen im Verlauf des 20. Jahrhunderts, darunter die veränderten Sozialisationsbedingungen, der Wunsch nach einer besseren Vereinbarkeit von Familie und Beruf sowie ein Anstieg in der Bedeutung schulischer Bildung prägen im deutschsprachigen Raum den Diskurs um den Inhalt von Bildung und die Bedeutung des Bildungssystems (Schüpbach, 2010). Dabei werden gerade Tagesschulen oftmals als mögliche Antwort auf wachsende Herausforderungen und Ansprüche betrachtet, was sich auch in den vielfältigen Begründungen des Tagesschulausbaus zeigt (Holtappels \& Rollett, 2009): Erstens werden Tagesschulen als wertvoller Beitrag zur soziokulturellen Infrastruktur gesehen, welcher die Erwerbstätigkeit der Eltern erleichtert und auch den Kindern eine gesellschaftliche und bildungsbezogene Teilhabe ermöglicht. Zweitens sollen Tagesschulen dem zunehmenden Bedürfnis nach ausserfamilialer und institutionalisierter Sozialisation gerecht werden und zu sozialer Integration beitragen. Drittens erhofft man sich durch ausserunterrichtliche Aktivitäten eine veränderte und erweiterte Lernkultur mit differenzierten Lerngelegenheit, durch die alle Schülerinnen und Schüler erreicht und gefördert werden. Diese Erwartung hinsichtlich einer verbesserten schulischen Förderung bezieht sich insbesondere auf Schülerinnen und Schüler, die während und zum Schluss ihrer Bildungslaufbahn nur unzureichende schulische Leistungen vorweisen können. Von diesem Schulversagen sind Kinder aus Familien mit niedrigem sozioöko- 
nomischem Status und mit Migrationshintergrund überdurchschnittlich häufig betroffen, weshalb man sich durch die erweiterten Lerngelegenheiten im Tagesschulangebot viertens einen Abbau von herkunftsbedingter Chancenungleichheit im Bildungssystem erhofft.

\section{Tagesschulqual ität}

Der fortgeschrittene Ausbau von Tagesschulen und Ganztagsschulen sowie die damit verbundenen Erwartungen führten im letzten Jahrzehnt zu einer Intensivierung der wissenschaftlichen Auseinandersetzung mit deren Qualität. Während in Deutschland insbesondere die Studie zur Entwicklung von Ganztagsschulen $[S t E G]$ zu einer immensen Datensammlung und zahlreichen Veröffentlichungen führte (z.B. Fischer et al., 2011), wurden in der Deutschschweiz das vom Schweizerischen Nationalfonds finanzierte Forschungsprojekt EduCare - Qualität und Wirksamkeit der familialen und ausserfamilialen Bildung und Betreuung im Primarschulalter (Schüpbach, 2010) sowie dessen Nachfolgeprojekt EduCare-TaSe - Tagesschulen und Schulerfolg lanciert.

Wenn es darum geht, Qualitätskriterien für Tagesschulen oder Ganztagsschulen zu bestimmen, lassen sich zwei Perspektiven unterscheiden (Holtappels, 2009). Einerseits beschreibt Holtappels eine systematisch-normative Perspektive, die sich an sozialisations-, bildungs- und schultheoretischen Begründungen orientiert. Nebst normativen Bestimmungen, ab wann man überhaupt von einer Tagesschule spricht, geht es hier also primär darum, welche Funktionen und Aufgaben von Tagesschulen zu erfüllen sind. Im Gegensatz dazu bezieht sich die empirische Perspektive auf "theoriegeleitete und empirisch fundierte Erkenntnisse über Zusammenhänge von Qualitätsmerkmalen, Bedingungen und Wirkungen» (Holtappels, 2009, S. 11). Die beiden Perspektiven schliessen sich aber keineswegs aus, so bedürfen systematisch-normative Festlegungen einer "durch empirische Erkenntnisse fundierten Schultheorie» (Holtappels, 2009, S. 12) und auch empirisch abgestützte Qualitätsmodelle nehmen immer gewisse normative Haltungen ein, insbesondere in Bezug auf die angestrebten Wirkungen.

Übersichtsarbeiten zu gängigen Qualitätsmodellen von ausserunterrichtlichen Angeboten an Tagesschulen oder Ganztagsschulen offenbaren eine Vielfalt an Dimensionen und Strukturen, die sich zum Teil auch in den verwendeten Begrifflichkeiten und deren Bedeutung stark unterscheiden (z.B. Fischer et al., 2011; Holtappels, 2009; Schüpbach, 2010). Gemeinsam ist den meisten Modellen, dass im Sinne des CIPO-Modells (Context Input Process Output oder auch CIPP-Modell; Stufflebeam, 1972) zwischen einer Input- bzw. Kontextebene, einer Prozessebene und einer Output- bzw. Wirkungsebene unterschieden wird. Häufig wird auch dem Umstand Rechnung getragen, dass sich Qualitätsmerkmale auf das Bildungssystem, auf die Einzelschule, auf Klassen oder auf 
einzelne Kinder beziehen können. Ausgehend von Angebots-Nutzungs-Modellen (z.B. Helmke, 2003) berücksichtigen neuere Modelle zudem stärker, dass die Wirkung der Angebote auch von der Nutzung der einzelnen Schülerinnen und Schüler abhängt.

\section{Theoretisches Rahmenmodell zur Erklärung der Ausbauqual ität}

Forschungsbemühungen zur Qualität von Tagesschulen lassen sich je nach Fragestellung stärker der Schuleffektivitätsforschung oder der Schulentwicklungsforschung zuordnen: Gemäss Fischer, Radisch, Theis und Züchner (2012) befasst sich die Schuleffektivitätsforschung mit den Bedingungen "guter Schulen» im Hinblick auf schulische Lernergebnisse, während die Schulentwicklungsforschung Schulqualität bezüglich deren Entwicklungsbedingungen untersucht. Ein Qualitätsmodell zu letzterem Forschungszweig unterscheidet drei Bedingungsfelder, welche die Ausbauqualität des Ganztagsbetriebs beeinflussen (Holtappels \& Rollett, 2008, 2009, Abs. 1). Da dieses Modell für die in diesem Artikel fokussierten Fragestellungen den stärksten Detaillierungsgrad aufweist, wird im Folgenden kurz auf die einzelnen Aspekte eingegangen.

Das theoretische «Rahmenmodell zur Erklärung der Ausbauqualität des Ganztagsbetriebes» (Holtappels \& Rollett, 2008, 2009) bildet den theoretischen Wirkungszusammenhang zwischen verschiedenen Schulentwicklungsmerkmalen und der Ausbauqualität der ausserunterrichtlichen Angebote einer Ganztagsschule ab, wobei es sich auf empirische Ergebnisse der Innovations- und Schulentwicklungsforschung abstützt (einen Überblick dazu bieten z.B. Rollett \& Holtappels, 2010). Unter "Ausbauqualität» wird dabei «das Ergebnis eines mehr oder minder gelungenen Ganztagsschulausbaus» (Holtappels \& Rollett, 2009, S. 22) verstanden, wobei hier ausgehend von bildungs- und schultheoretischen Begründungen des Ausbaus eine normative Wertung vorgenommen wird. Dazu gehören beispielsweise Umfang und Breite des Angebots, zeitlicher Umfang des Betriebes, Umfang der Schülerteilnahme am Angebot oder auch die Zufriedenheit der beteiligten Gruppen mit demselben. Als zentrale Bedingungsfelder der Ausbauqualität unterscheiden Holtappels und Rollett zwischen «Ziele und Konzeption», "Organisationskultur» sowie dem "Entwicklungsprozess»: Der Qualitätsbereich "Ziele und Konzeption» bezieht sich auf die Ziele, welche mit dem Angebot verfolgt werden, worunter auch die Gründungsmotive hinter dem Ausbau fallen, sowie den erreichten konzeptionellen Entwicklungsstand. Unter «Organisationskultur» werden insbesondere das Gelingen inner- und ausserschulischer Kooperation, die Innovationsbereitschaft und die Beteiligung verschiedener Personengruppen am Ganztagsbetrieb zusammengefasst. Der eigentliche "Entwicklungsprozess», welcher dem Ganztagsausbau zugrunde liegt, wird als eigener Qualitätsbereich angesehen, mit welchem unter anderem die Art 
und der Umfang von Entwicklungsmassnahmen sowie die Beteiligung interner und externer Gruppen oder Institutionen am Entwicklungsprozess gemeint sind. Im Hinblick auf die zuvor beschriebenen Qualitätsmodelle bleibt festzuhalten, dass mit dem Rahmenmodell von Holtappels und Rollett $(2008,2009)$ nur ein Ausschnitt der Tagesschulqualität abgebildet werden kann. So bleiben beispielsweise nationale oder kantonale Vorgaben, ausserschulische Akteure oder auch die Wirkungsebene unberücksichtigt.

\section{Forschungsstand}

Die genannten Erwartungen an eine erweiterte Lernkultur und mehr Bildungsgleichheit stellen klare Anforderungen an ein vielfältiges Förderangebot in Tagesschulen, in welchem individuelle Bedürfnisse der einzelnen Schülerinnen und Schüler berücksichtigt werden. Ob diese Anforderungen erfüllt werden, ist für die Deutschschweiz allerdings weitgehend unklar. Im Hinblick auf gesetzliche Regulierungen bestehen auf nationaler Ebene keinerlei pädagogische Vorgaben für Tagesschulen und auf kantonaler Ebene zeigen sich deutliche Unterschiede, ob und inwieweit solche Anforderungen existieren (Schultheiss \& Stern, 2013). Es ist denn auch nicht erstaunlich, wenn in Bezug auf Tagesschulen und deren Qualitätskriterien eine gewisse Konzeptlosigkeit konstatiert wird (Flitner, 2011). Im Folgenden wird ein kurzer Überblick über den bisherigen Forschungsstand zum Ausbau von Tagesschulen respektive von Ganztagsschulen gegeben. Für weitere Befunde aus der Innovations- und Schulentwicklungsforschung, welche auch dem theoretischen Rahmenmodell von Holtappels und Rollett (2008, 2009) zugrunde liegen, verweisen wir an dieser Stelle auf den Überblick bei Rollett \& Holtappels (2010).

Bisher fehlen für die Deutschschweiz Befunde zu den Motiven hinter dem Ausbau von Tagesschulen und zur Beteiligung unterschiedlicher Professionsgruppen an der Ausarbeitung von Konzepten. Eine bessere Datenlage präsentiert sich in Deutschland durch eine repräsentative Befragung im Rahmen von StEG (Holtappels, 2008): So zeigte sich, dass insbesondere die Verbesserung von Bildungschancen und der individuellen Förderung zentrale Motive für den Ausbau darstellten, während der soziale Bedarf im Stadtteil und insbesondere der Wunsch des Lehrerkollegiums weniger ausschlaggebend waren. Nach eigener Angabe der Lehrkräfte waren diese in 61\% der Fälle zumindest teilweise an der Erarbeitung des Ganztagsschulkonzepts beteiligt. Insgesamt war die Lehrerpartizipation an Entwicklungsarbeiten in Grundschulen deutlich höher als in Sekundarschulen und in offenen Ganztagsschulen niedriger als in gebundenen Ganztagsschulen.

Informationen zur Häufigkeit von individualisierenden und differenzierenden Fördermassnahmen an Deutschschweizer Tagesschulangeboten sind bisher ebenfalls kaum vorhanden. Schüpbach (2010) konnte aufzeigen, dass 
"Hausaufgabenhilfe und -betreuung» in Deutschschweizer Tagesschulen signifikant häufiger vorkommt als in anderen Schulformen. Dabei wurde allerdings auf eine Unterscheidung offener und gebundener Tagesschulen verzichtet und die einzelnen Förderangebote sind nicht weiter differenziert dargestellt. Für Deutschland zeigte die erste $S t E G$-Erhebungswelle, dass im Jahr 2009 nahezu sämtliche Ganztagsschulen in Deutschland Hausaufgabenhilfe oder zumindest -betreuung angeboten haben. Etwas seltener wurden Förderunterricht für Schülerinnen und Schüler mit über- oder unterdurchschnittlich guten Fachleistungen (85\%) und spezifische Fördermassnahmen, z.B. für Schülerinnen und Schüler nichtdeutscher Muttersprache oder Herkunft (61\%) angeboten (Rollett, Lossen, Jarsinski, Lüpschen \& Holtappels, 2011). Auch für das Jahr 2012 zeigte die zweite StEG-Erhebungswelle, dass lernunterstützende Elemente in ausserunterrichtlichen Ganztagsangeboten in Deutschland weit verbreitet sind und dass dies auch für den Primarbereich gilt (Fischer, Klieme, Holtappels, Stecher \& Rauschenbach, 2013). Leider wurde bei diesen Ergebnissen nicht zwischen offenen und gebundenen Ganztagsschulen unterschieden, wobei gebundene Modelle einen grösseren Handlungsspielraum durch Schulleitung und Lehrpersonen bieten, z.B. hinsichtlich der Rhythmisierung von unterrichtlichen und ausserunterrichtlichen Elementen. Der hohe Anteil offener Ganztagsschulen (80\% im Jahr 2012) deutet immerhin darauf hin, dass Hausaufgabenbetreuung und weitere Förderangebote auch von der Mehrzahl offener Ganztagsschulen angeboten werden.

Erste empirische Analysen zur Überprüfung des Rahmenmodells zur «Ausbauqualität des Ganztagbetriebes» wurden im Rahmen des Forschungsprojekts StEG durchgeführt (Holtappels \& Rollett, 2008; Rollett \& Holtappels, 2010; Rollett et al., 2011). Spezifische Ergebnisse zu individualisierenden und differenzierenden Förderangeboten an Ganztagsschulen fehlen bisher, dafür wurden Breite und Umfang der Angebotsstruktur untersucht. Dabei zeigte sich für den Grundschulbereich, dass intensivere Entwicklungsbemühungen, eine stärkere Beteiligung der Lehrkräfte in den Ganztagsangeboten und eine flexiblere Zeitorganisation die Entwicklung von Angebotsbreite und -umfang positiv beeinflussten. Im Gegensatz dazu zeigte eine bessere Zusammenarbeit zwischen Lehrkräften und pädagogisch tätigem Personal einen leicht hemmenden Einfluss auf die Angebotsbreite, was mit einer möglicherweise stärker vorhandenen Fokussierung auf gewisse Angebotsmerkmale begründet wurde (Rollett et al., 2011). Für die Entwicklung der Angebotsbreite im Sekundarschulbereich zeigten stärker ausgeprägte pädagogische Entwicklungsziele und umfangreichere konzeptuellen Festlegungen einen positiven Effekt. Dasselbe gilt für die Innovationsbereitschaft und Zusammenarbeit in den Lehrerkollegien sowie eine stärkere Beteiligung der Lehrkräfte in den Ganztagsangeboten. Systematische Qualitätsentwicklung, externe Unterstützungsmassnahmen sowie eine intensivere Weiterentwicklung des Ganztagsschulkonzepts wirkten sich ebenfalls förderlich auf Angebotsbreite und -umfang aus (Rollett et al., 2011). 


\section{Fragestellungen}

Ausgehend vom bisherigen Forschungsstand lassen sich folgende Fragestellungen skizzieren, die im Weiteren untersucht werden:

(1) Welche Rolle spielten pädagogische Motive bei der Tagesschulgründung?

(2) Wie intensiv waren Schulleitung und Lehrpersonen an der Erarbeitung von Leitlinien zum Tagesschulangebot beteiligt?

(3) Wie stark sind Hausaufgabenhilfe und -betreuung sowie stärker differenzierende Förderangebote in den Tagesschulangeboten vertreten?

(4) Können Unterschiede in der Verfügbarkeit solcher Förderangebote durch pädagogische Motive, konzeptionelle Beteiligung der Schulleitung oder der Lehrpersonen und durch konzeptionelle Festlegungen zur Organisation der Tagesschulangebote erklärt werden?

\section{Methode}

\section{Stichprobenziehung}

Die Daten für die nachfolgenden Analysen stammen vom laufenden Forschungsprojekt EduCare-TaSe - Tagesschulen und Schulerfolg?, welches durch den Schweizerischen Nationalfonds finanziert wird. Im Rahmen des Forschungsprojekts wurde eine Vollerhebung bei offenen Deutschschweizer Tagesschulen im Primarschulbereich durchgeführt, wobei aus forschungsökonomischen Gründen mindestens zwei parallele Erstjahrgangsklassen vorausgesetzt wurden. Ausgehend von der Definition der kantonalen Erziehungsdirektoren (EDK, 2013) wurde folgende Operationalisierung vorgenommen: Eine offene Tagesschule ist eine Schule, die ein modular aufgebautes und freiwilliges ausserunterrichtliches Bildungs- und Betreuungsangebot anbietet, welches an mindestens drei Tagen die Woche jeweils am Mittag sowie am Nachmittag zur Verfügung steht. Von den insgesamt 251 angefragten offenen Tagesschulen erklärten sich 53 bereit, an der Studie teilzunehmen, wodurch 13 Deutschschweizer Kantone vertreten sind. Da bei einer Tagesschule die Angaben zu den Förderangeboten fehlen, beschränkt sich dieser Artikel auf die verbleibenden 52 Tagesschulen.

\section{Datenerhebung}

Die Daten für den vorliegenden Artikel wurden zwischen Dezember 2013 und September 2014 bei den 52 Schul- und Tagesschulangebotsleitungen via online-Fragebogen erhoben. Dabei wurden Angaben zu verschiedenen Förderangeboten, zu pädagogischen Motiven hinter dem Tagesschulausbau, zu konzeptionellen Festlegungen in den Leitlinien der Tagesschulangebote und zur Beteiligung verschiedener Professionsgruppen an der konzeptionellen Arbeit erfragt. Dazu orientierten wir uns hauptsächlich an Messinstrumenten, welche bereits bei $S t E G$ eingesetzt wurden (Quellenberg, 2009), wobei wir die Fragen an den Schweizerischen Sprach- und Kulturraum adaptierten. 
Pädagogische Motive bei der Tagesschulgründung

Mittels Befragung der Schulleitungen wurde erhoben, inwieweit pädagogische Motive («Sozialer Bedarf in der Gemeinde», «Wunsch der unterrichtenden Lehrpersonen», "Verbesserung der Bildungschancen» und «Verbesserung der individuellen Förderung»; adaptiert von Quellenberg, 2009) zur Umwandlung der Schule in eine Tagesschule führten. Dazu mussten die vier Items auf einer vierstufigen Skala $(0=$ "Trifft gar nicht zu», 1 = "Trifft eher nicht zu», $2=$ "Trifft eher zu» und 3 = "Trifft voll und ganz zu») beurteilt werden. Die interne Konsistenz der Skala "pädagogische Motive» ist akzeptabel $(\alpha=0.74)$ und vergleichbar mit den Werten bei Quellenberg $(\alpha=0.78)$.

\section{Konzeptionelle Festlegungen zur Organisation}

Über die Tagesschulangebotsleitungen wurde das Ausmaß konzeptioneller Festlegungen zur Organisation der Tagesschulangebote erfasst (adaptiert von Quellenberg, 2009). Auf einer vierstufigen Skala (0 = "Gar nicht», 1 = "Ansatzweise», 2 = "Weitgehend» und 3 = "Umfassend») wurde beurteilt, inwieweit in den Leitlinien der Tagesschulangebote Aussagen zu neun verschiedenen Arbeitsfeldern ("Zeitorganisation und Rhythmisierung», "Schulräumliche Organisation und Gestaltung», "Elternmitarbeit bzw. Kooperation mit Eltern», etc.) gemacht wurden. Die Skala erzielte eine gute interne Konsistenz $(\alpha=0.86)$, was in etwa dem Wert bei Quellenberg entspricht $(\alpha=0.80)$.

\section{Partizipation an (Weiter-) Entwicklung der Leitlinien} Ebenfalls über die Tagesschulangebotsleitungen wurde für verschiedene Professionsgruppen erfragt, inwieweit diese an der (Weiter-) Entwicklung der Leitlinien zum Tagesschulangebot beteiligt waren $(0=$ "Gar nicht», $1=$ «In geringem Umfang», 2 = "In mässigem Umfang» und 3 = "In hohem Umfang»; adaptiert von Quellenberg, 2009). Da die Items nur eine geringe interne Konsistenz aufweisen $(\alpha=0.34)$ und sich auch nicht für eine Faktorenanalyse eignen $(\mathrm{KMO}=0.46$; Hutcheson \& Sofroniou, 1999), wurden keine Skalen gebildet. Stattdessen wurden für weitere Analysen die Einzelitems zur Partizipation durch die Schulleitung und zur Partizipation durch die Lehrpersonen verwendet, wobei letzteres aufgrund einzelner Ausreisser zusätzlich dichotomisiert $0=$ «keine Partizipation», $1=$ «Partizipation»; $\mathrm{M}=0.25, \mathrm{SD}=0.44$ wurde.

\section{Förderangebote}

Schliesslich wurde die Tagesschulangebotsleitung auch noch zu möglichen Förderangeboten im Tagesschulangebot befragt (adaptiert von Quellenberg, 2009). Dabei sollten die Tagesschulangebotsleiterinnen und -leiter auf einer sechsstufigen Skala $(0=$ "Nein», 1 = "Ja, monatlich», $2=$ "Ja, mehrmals im Monat», 3 = «Ja, wöchentlich», $4=$ = Ja, mehrmals die Woche» und $5=$ "Ja, täglich») festhalten, ob und wie häufig verschiedene Förderangebote («Hausaufgabenhilfe», «Hausaufgabenbetreuung», «Förderung von Kindern mit schwachen 
Schulleistungen», «Förderung von Kindern mit sehr guten Schulleistungen» und «Förderung von Kindern mit nichtdeutscher Muttersprache») in den Tagesschulangeboten verfügbar waren.

\section{Alter der Tagesschule}

Um einen entsprechenden Einfluss zu kontrollieren, wurden die Schulleitungen zusätzlich danach befragt, seit wie vielen Jahren Tagesschulangebote geführt werden $(\mathrm{M}=7.90, \mathrm{SD}=7.89)$.

\section{Ergebnisse}

\section{Deskriptive Ergebnisse zu den Schulentwicklungs- merkmalen}

In Tabelle 1 sind die deskriptiven Ergebnisse zu den drei untersuchten Schulentwicklungsmerkmalen ersichtlich. Zusätzlich sind in Tabelle 2 die Antworthäufigkeiten zu den einzelnen pädagogischen Motiven und der konzeptionellen Partizipation dargestellt. Unter den erfragten pädagogischen Motiven für die Tagesschulgründung nahm der soziale Bedarf in der Gemeinde die zentralste Rolle ein: $82.7 \%(M=2.23)$ der Schulleitungen stimmten zu, dass dieses Motiv zumindest teilweise für den Ausbau der Schule in eine Tagesschule verantwortlich war. Die Motive «Verbesserung der Bildungschancen» (48.1\%, $\mathrm{M}=1.35$ ), "Verbesserung der individuellen Förderung» $(25 \%, M=1.02)$ und "Wunsch der unterrichtenden Lehrpersonen» $(25 \%, \mathrm{M}=0.85)$ fanden hingegen deutlich weniger Zustimmung. Bezüglich der Ausarbeitung oder Weiterentwicklung von Leitlinien zum Tagesschulangebot wurde angegeben, dass die Schulleitung in $67.3 \%$ der Fälle zumindest teilweise und zu 32.7\% in hohem Umfang beteiligt war. Im Gegensatz dazu beteiligten sich nur 25\% der Lehrpersonen zumindest teilweise und lediglich 5.8\% in hohem Umfang.

\section{Deskriptive Ergebnisse und Clusteranalyse zu} Förderangeboten

Tabelle 3 zeigt die Verfügbarkeit der erfragten Förderangebote in den untersuchten Tagesschulen. Nahezu sämtliche Tagesschulen bieten täglich oder zumindest mehrmals die Woche Hausaufgabenbetreuung an. Konkrete Hausaufgabenhilfe steht hingegen noch in rund 50\% der Tagesschulen zur Verfügung und Förderangebote für bestimmte Schülerinnen und Schüler sind selten. Um mögliche Muster zu identifizieren, wurde mit den fünf Items eine hierarchische Clusteranalyse nach Ward durchgeführt. Eine Visualisierung des Anstiegs in der Fehlerquadratsumme mittels Dendrogramm und Struktogramm sprach deutlich für eine Lösung mit zwei Clustern. Mit den dadurch ermittelten Clusterzentren wurde im Anschluss eine nichthierarchische Clusteranalyse (k-means) durchgeführt, wodurch vier Fälle (7.7\%) das Cluster wechselten. Diese Lösung konnte 
durch eine Diskriminanzanalyse bestätigt werden, wobei 98.1\% der Fälle richtig klassifiziert wurden und schliesslich führte auch eine Kreuzvalidierung in 96.2\% der Fälle zu einer korrekten Zuordnung.

Aus Abbildung 2 ist ersichtlich, dass nahezu sämtliche Tagesschulangebote, unabhängig von der Gruppenzugehörigkeit, täglich oder mehrmals wöchentlich die Möglichkeit zur betreuten Bearbeitung von Hausaufgaben anbieten. Deutliche Unterschiede zeigen sich allerdings bezüglich der Verfügbarkeit von weiteren Förderangeboten: Hausaufgabenhilfe (z.B. durch die Lehrperson) steht im Cluster 1 nur in 37.1\% der Tagesschulangebote zur Verfügung und differenzierende Förderangebote für leistungsschwache, leistungsstarke oder fremdsprachige Schülerinnen und Schüler fehlen fast gänzlich (jeweils in höchstens $11.4 \%$ der Tagesschulangebote vorhanden). Im Gegensatz dazu bieten 88.2\% der Tagesschulangebote im Cluster 2 eine Hausaufgabenhilfe an und auch die differenzierenden Förderangebote sind jeweils in mindestens $70.6 \%$ der Tagesschulangebote anzutreffen. Während sich Tagesschulen im Cluster 1 also vorwiegend auf Hausaufgabenbetreuung beschränken, verfügen Tagesschulen im Cluster 2 über ein breiteres Förderangebot. Im nächsten Schritt soll anhand einer logistischen Regressionsanalyse untersucht werden, ob die Clusterzugehörigkeit mittels Schulentwicklungsmerkmalen vorhergesagt werden kann.

\section{Logistische Regressionsanalyse zur Vorhersage der Verfügbarkeit von Förderangeboten}

Um mögliche Wirkungen der beschriebenen Schulentwicklungsmerkmale auf die Verfügbarkeit von individualisierenden und differenzierenden Förderangeboten $\mathrm{zu}$ untersuchen, wurde schrittweise eine logistische Regressionsanalyse gerechnet, mit der Clusterzugehörigkeit $(0=$ "Hausaufgabenbetreuung»; $1=$ «Hausaufgabenbetreuung und weitere Förderangebote») als zu erklärende Variable (Tabelle 4). In einem ersten Schritt wurden die pädagogischen Motive in das Modell aufgenommen $\left(C h i^{2}=1.77, p=0.18\right)$, wobei diese keinen signifikanten Einfluss auf die Clusterzugehörigkeit zeigten $(p=0.20)$. Im zweiten Schritt wurde das Ausmass konzeptueller Festlegungen zur Organisation der Tagesschulangebote aufgenommen, wodurch ein signifikanter Anteil der Varianz erklärt werden konnte $\left(\Delta \mathrm{Chi}^{2}=6.28, p<0.05\right)$ : Umfassendere konzeptuelle Festlegungen erhöhten die Wahrscheinlichkeit, nebst Hausaufgabenbetreuung auch noch weitere Förderangebote vorzufinden $(\operatorname{Exp}(b)=3.36, p<0.05)$. Im dritten Schritt wurde die Partizipation durch Schulleitung und Lehrpersonen an der Ausarbeitung der Leitlinien zum Tagesschulangebot in das Modell aufgenommen. Weder die Beteiligung der Schulleitung $(p=0.14)$ noch der Lehrpersonen $(p=0.21)$ zeigte einen signifikanten Einfluss auf die Clusterzugehörigkeit, gemeinsam leisteten die beiden Items aber einen signifikanten Beitrag zur erklärten Varianz $\left(\Delta C h i^{2}=8.05, \mathrm{p}<0.05\right)$. Das Ausmass konzeptueller Festlegungen zur Organisation der Tagesschulangebote zeigte nach wie vor einen signifikanten Einfluss auf die Clusterzugehörigkeit $(\operatorname{Exp}(b)=4.97, p<0.01)$. 
Insgesamt konnte dieses Modell 37\% der Varianz in der Verfügbarkeit von individualisierenden und differenzierenden Förderangeboten erklären. Im vierten und letzten Schritt wurde schliesslich das Alter der Tagesschule als Kontrollvariable hinzugefügt, welches allerdings keinen Einfluss auf die Clusterzugehörigkeit zeigte $(p=0.12)$ und das Modell nicht signifikant veränderte $(\Delta$ $\left.\mathrm{Chi}^{2}=3.48, p=0.06\right)$.

\section{Diskussion}

Unsere Studie untersuchte anhand einer Stichprobe von offenen Tagesschulen, (1) welche Rolle pädagogische Motive bei der Tagesschulgründung spielten, (2) wie intensiv Schulleitung und Lehrpersonen an der Erarbeitung von Leitlinien zum Tagesschulangebot beteiligt waren, (3) wie stark Hausaufgabenhilfe und -betreuung sowie stärker differenzierende Förderangebote in den Tagesschulangeboten vertreten sind und (4) ob Unterschiede in der Verfügbarkeit solcher Förderangebote durch ausgewählte Schulentwicklungsmerkmale erklärt werden können.

\section{Pädagogische Motive \& konzeptionelle Beteiligung}

Die Ergebnisse zu den pädagogischen Motiven zeigen, dass der Ausbau offener Tagesschulen in der Deutschschweiz ganz im Gegensatz zu Deutschland (Holtappels, 2008) nur selten durch eine erhoffte Verbesserung der Bildungschancen oder der individuellen Förderung angeregt wurde und dass auch der Wunsch der unterrichtenden Lehrpersonen nur beim Ausbau weniger Tagesschulen eine Rolle spielte. Deutlich ausschlaggebender scheint hingegen der soziale Bedarf in der jeweiligen Gemeinde zu sein. Unsere Befunde zu den untersuchten Motiven verdichten demnach den Eindruck, dass bildungsbezogene Überlegungen für den gegenwärtigen Ausbau von Tagesschulen in der Deutschschweiz nur eine untergeordnete Rolle spielen und dass Tagesschulen primär eine Antwort auf die gewachsene Nachfrage nach ausserfamiliären Betreuungsformen durch Familien und durch die Wirtschaft darstellen. Vor diesem Hintergrund ist auch nicht weiter erstaunlich, dass Lehrpersonen nur bei 25\% der Tagesschulen zumindest teilweise an der Ausarbeitung von Leitlinien zum Tagesschulangebot beteiligt waren und dass selbst die Schulleitung zu einem Drittel überhaupt nicht involviert war.

\section{Individualisierende und differenzierende}

Förderangebote

Während fast alle untersuchten Tagesschulen Hausaufgabenbetreuung anbieten, sind konkrete Hausaufgabenhilfen und insbesondere Förderangebote für bestimmte Schülerinnen und Schüler, auch im Vergleich zu den Ganztagsschulen in Deutschland (z.B. Fischer et al., 2013), eher selten anzutreffen. 
Eine Clusteranalyse zeigte auf, dass sich etwa zwei Drittel der Tagesschulen fast ausschliesslich auf Hausaufgabenbetreuung beschränken, während das Angebot bei den restlichen Tagesschulen deutlich vielfältiger ausfällt. Insbesondere die differenzierenden Förderangebote für leistungsschwache, leistungsstarke oder fremdsprachige Schülerinnen und Schüler werden demnach selten alleine, sondern meist in Kombination mit weiteren Förderelementen angeboten.

\section{Einfluss von Schulentwicklungsmerkmalen auf die Verfügbarkeit von Förderangeboten}

Ausgehend vom «Rahmenmodell zur Erklärung der Ausbauqualität des Ganztagsbetriebes» (Holtappels \& Rollett, 2008) haben wir untersucht, ob ein vielfältigeres Förderangebot durch ausgewählte Schulentwicklungsmerkmale vorhergesagt werden kann. Dabei zeigte sich erstens, dass insbesondere Tagesschulangebote mit umfassenderen konzeptuellen Festlegungen über eine breitere Palette an Förderangeboten verfügen, was sich mit den Befunden von Rollett et al. (2011) zur Angebotsbreite an deutschen Ganztagsschulen im Sekundarschulbereich deckt. Dass eine konzeptuelle Verankerung der Gestaltungselemente, der Zeitorganisation oder der Verknüpfung von Unterricht und Tagesschulangebot zusätzliche Förderangebote anregen kann, ist naheliegend. Möglich wäre aber auch, dass die Förderangebote zu neuen konzeptuellen Festlegungen führen, z.B. wenn dadurch erstmals eine Kooperation zwischen Lehrpersonal und weiterem pädagogisch tätigem Personal stattfindet.

Zweitens zeigte die Beteiligung durch Lehrpersonen und Schulleitung bei der Ausarbeitung von Leitlinien zum Tagesschulangebot einen Einfluss auf die Verfügbarkeit von Förderangeboten. Für sich genommen wirkte sich allerdings weder die Partizipation der Lehrpersonen noch der Schulleitung auf die Verfügbarkeit der Förderangebote aus. Dieser Befund könnte ein Ergebnis unserer kleinen Stichprobe und der damit verbundenen geringen Teststärke sein, könnte aber auch auf ein komplexeres Zusammenspiel zwischen der Beteiligung der beiden Professionsgruppen hinweisen.

Drittens konnten wir keinen Einfluss der pädagogischen Motive hinter dem Tagesschulausbau auf die Verfügbarkeit von Förderangeboten feststellen. Dies ist insofern erstaunlich, als dass gerade die Motive «bessere Bildungschancen» und «bessere individuelle Förderung» einen konkreten Bezug zu den untersuchten Förderangeboten haben und bei StEG ein positiver Einfluss von stärker ausgeprägten pädagogischen Entwicklungszielen auf die Entwicklung der Angebotsbreite festgestellt wurde (Rollett et al., 2011). Allerdings scheinen diese beiden Motive bei nahezu sämtlichen Tagesschulen nur eine untergeordnete Rolle zu spielen, was den fehlenden Zusammenhang erklären könnte. Alternativ wäre es aber auch möglich, dass sich die Motive hinter der Tagesschulgründung nicht auf die konkreten Ziele und deren Umsetzung in den Tagesschulangeboten auswirken. 
Insbesondere die Analyse zum Einfluss der Schulentwicklungsmerkmale auf die Verfügbarkeit von Förderangeboten ist mit einigen Einschränkungen verbunden: So konnten wir (1) nicht sämtliche Bereiche abdecken, die gemäss Rahmenmodell von Holtappels und Rollett (2008) von Bedeutung sind, was Erkenntnisse über die genauen Wirkmechanismen erschwert. Ein Grund dafür liegt im bereits sehr umfangreichen Fragenkatalog, bei dem wir uns auf ausgewählte Aspekte von Schulentwicklungsmerkmalen beschränken mussten. Ein zweiter Grund stellt die Stichprobe dar, die bereits für die vorliegende Regressionsanalyse eher klein war und mit weiteren Items an ihre Grenze gestossen wäre. Als weitere Einschränkung ist denn auch (2) die kleine Stichprobe zu nennen. So war die Teststärke eher gering und die einzelnen Tagesschulen hatten einen starken Einfluss auf die gerechneten Modelle, was sich auch an teils auffälligen Residuen zeigte. Für die gefundenen Effekte spricht allerdings, dass sich diese auch unter Ausschluss von Ausreissern als einigermassen stabil erwiesen. Wie bereits angesprochen, erlaubt unser Beitrag (3) keine abschliessenden Aussagen zur Kausalität, da Förderangebote und Entwicklungsmerkmale im Querschnitt erhoben wurden und es sich bei den pädagogischen Motiven und der konzeptionellen Beteiligung nur um retrospektive Einschätzungen handelt - im Fall der konzeptionellen Beteiligung durch Lehrpersonen und Schulleitung gar um eine Fremdeinschätzung. (4) Schliesslich ist festzuhalten, dass unserer Analyse hinsichtlich der Verfügbarkeit von Förderangeboten nur einen Teilaspekt der Tagesschulqualität abdeckt und weitere Qualitätsmerkmale unberücksichtigt bleiben.

\section{Faz it}

Im gegenwärtigen Diskurs um Bildung nehmen Tagesschulen eine zentrale Rolle ein, wobei auch Hoffnungen hinsichtlich mehr Bildungsgerechtigkeit und einer verbesserten individuellen Förderung geäussert werden. Bezogen auf offene Tagesschulen aus der Deutschschweiz zeigen unsere Ergebnisse allerdings, dass bildungsbezogene pädagogische Motive beim Ausbau von Tagesschulangeboten nur selten von Bedeutung sind und dass sich gerade Lehrpersonen kaum an der konzeptionellen Ausarbeitung beteiligen. Zwar bieten nahezu sämtliche untersuchten Tagesschulangebote Hausaufgabenbetreuung an, darüber hinaus sind individualisierende und differenzierende Förderangebote allerdings nur bei etwa einem Drittel der Tagesschulen vorhanden. Ob solche Förderangebote zur Verfügung stehen und dadurch im Sinne aktueller Qualitätsmodellen eine Zunahme von Bildungserfolg und -gerechtigkeit erwartet werden kann, dürfte einerseits von konzeptionellen Festlegungen zur Organisation der Tagesschulangebote und andererseits von der Beteiligung durch Lehrpersonen und Schulleitung bei der Ausarbeitung von Leitlinien abhängen. 


\section{Li teraturverzeichnis}

EDK (2013). Kantonsumfrage 2012/2013. Zugriff am 14.09.2015-unter http://edudoc.ch/ record/115194/files/2013.pdf

Fischer, N., Holtappels, H. G., Klieme, E., Rauschenbach, T., Stecher, L. \& Züchner, I. (2011). Ganztagsschule: Entwicklung, Qualität, Wirkungen. Längsschnittliche Befunde der Studie zur Entwicklung von Ganztagsschulen (StEG). Weinheim \& Basel: Beltz Juventa.

Fischer, N., Klieme, E., Holtappels, H. G., Stecher, L. \& Rauschenbach, T. (2013). Ganztagsschule 2012/2013. Deskriptive Befunde einer bundesweiten Befragung. Frankfurt: DIPF/ IFS/JLU/DJI.

Fischer, N., Radisch, F., Theis, D. \& Züchner, I. (2012). Qualität von Ganztagsschulen Bedingungen, Wirkungen und Empfehlungen. Expertise für die SPD Bundestagsfraktion. Frankfurt am Main.

Flitner, C. (2011). Absturzgefährdet-schulische Tagesbetreuung in der Schweiz. Zürich: vpod. Helmke, A. (2003). Unterrichtsqualität erfassen, bewerten, verbessern. Seelze: Kallmeyer.

Holtappels, H. G. (2008). Ziele, Konzepte, Entwicklungsprozesse. In H. G. Holtappels (Hrsg.), Ganztagsschule in Deutschland. Ergebnisse der Ausgangserhebung der «Studie zur Entwicklung von Ganztagsschulen» (StEG) (2., korrigierte Aufl., S. 139-163). Weinheim: Juventa.

Holtappels, H. G. (2009). Qualitätsmodelle - Theorie und Konzeptionen. In I. Kamski, H. G. Holtappels \& T. Schnetzer (Hrsg.), Qualität von Ganztagsschule. Konzepte und Orientierungen für die Praxis (S. 11-25). Münster: Waxmann Verlag GmbH.

Holtappels, H. G. \& Rollett, W. (2008). Organisationskultur, Entwicklung und Ganztagsschulausbau. In H. G. Holtappels (Hrsg.), Ganztagsschule in Deutschland. Ergebnisse der Ausgangserhebung der «Studie zur Entwicklung von Ganztagsschulen» (StEG) (2., korrigierte Aufl., S. 209-226). Weinheim: Juventa.

Holtappels, H. G. \& Rollett, W. (2009). Schulentwicklung in Ganztagsschulen. Zur Bedeutung von Zielorientierungen und Konzeption für die Qualität des Bildungsangebots. In L. Stecher, C. Allemann-Ghionda, W. Helsper \& E. Klieme (Hrsg.), Ganztägige Bildung und Betreuung (Zeitschrift für Pädagogik, Beiheft, Bd. 54, S. 18-39). Weinheim u.a.: Beltz.

Hutcheson, G. \& Sofroniou, N. (1999). The multivariate social scientist. Introductory statistics using generalized linear models. London: Sage Publications.

Quellenberg, H. (2009). Studie zur Entwicklung von Ganztagsschulen (StEG) - ausgewählte Hintergrundvariablen, Skalen und Indices der ersten Erhebungswelle. Frankfurt am Main: Gesellschaft zur Förderung Pädagogischer Forschung [u.a.].

Rollett, W. \& Holtappels, H. G. (2010). Entwicklung von Ganztagsschulen in Deutschland. Analysen zum Ausbaustand der Ganztagsschullandschaft und zu Entwicklungsbedingungen der Schülerteilnahme. In N. Berkemeyer, W. Bos, H. G. Holtappels, N. McElvany $\&$ R. Schulz-Zander (Hrsg.), Jahrbuch der Schulentwicklung. Daten, Beispiele und Perspektiven (S. 99-129). Weinheim: Beltz.

Rollett, W., Lossen, K., Jarsinski, S., Lüpschen, N. \& Holtappels, H. G. (2011). Ausserunterrichtliche Angebotsstruktur an Ganztagsschulen. Entwicklungstrends und Entwicklungsbedingungen. In N. Fischer, H. G. Holtappels, E. Klieme, T. Rauschenbach, L. Stecher \& I. Züchner (Hrsg.), Ganztagsschule: Entwicklung, Qualität, Wirkungen. Längsschnittliche Befunde der Studie zur Entwicklung von Ganztagsschulen (StEG) (S. 76-96). Weinheim \& Basel: Beltz Juventa.

Schultheiss, A. \& Stern, S. (2013). Familienergänzende Kinderbetreuung im Schulbereich. Stand in den Kantonen. Zürich: INFRAS im Auftrag der schweizerischen Konferenz der kantonalen Erziehungsdirektoren (EDK).

Schüpbach, M. (2010). Ganztägige Bildung und Betreuung im Primarschulalter. Qualität und Wirksamkeit verschiedener Schulformen im Vergleich. Wiesbaden: VS Verlag für Sozialwissenschaften. 
Sekretariat der Ständigen Konferenz der Kultusminister der Länder in der Bundesrepublik Deutschland (2011). Definitionenkatalog zur Schulstatistik. Zugriff am 14.09.2015-unter http://www.kmk.org/fileadmin/pdf/Statistik/Defkat2011.pdf

Stern, S., Iten, R., Schwab, S., Felfe, C., Lechner, M. \& Thiemann, P. (2013). Familienergänzende Kinderbetreuung und Gleichstellung. Zürich, St. Gallen: INFRAS, Schweizerisches Institut für Empirische Wirtschaftsforschung, Universität St. Gallen.

Stufflebeam, D. L. (1972). Evaluation als Entscheidungshilfe. In C. Wulf (Hrsg.), Evaluation. Beschreibung und Bewertung von Unterricht, Curricula und Schulversuchen (S. 113-145). München: Piper.

Schlagworte: Tagesschulen, Förderangebote, Bildungsgerechtigkeit, Tagesschulqualität, Schulentwicklung

\section{Enjeux éducatifs des écoles à horaire continu: quelques offres d'encouragement réalisées en Suisse alémanique}

\section{Résumé}

La thématique des horaires scolaires continus est au cœur des discussions actuelles sur l'éducation, notamment par rapport à la justice scolaire et au soutien individualisé. Toutefois, on manque encore d'informations sur les opportunités réelles d'apprentissage offertes par les écoles à horaire continu de Suisse alémanique. Cet article met en discussion les attentes éducatives envers ces écoles à horaire continu ainsi que les modèles de qualité en vigueur. Il examine également, pour les écoles à horaire continu de Suisse alémanique, les motivations pédagogiques qui prévalent à leur fondation, la participation des directions d'école et celle des enseignants lors de leur création, les différents dispositifs de soutien individualisé et différencié proposés tout en analysant si ceux-ci sont influencés par les développements actuels dans le champ scolaire.

Mots-clés: Écoles à horaire continu, offres d'encouragement, justice scolaire, qualité des écoles à horaire continu, développement scolaire 


\section{Le sfide educative delle scuole a orario continuato: alcune proposte di promozione realizzate in Svizzera tedesca}

\section{Riassunto}

L'orario scolastico continuato é una tematica importante nelle discussioni attuali in campo educativo, centrata soprattutto sull'equità scolastica e il sostegno individuale. Malgrado ciò, mancano ancora oggi le informazioni sulle reali opportunità d'apprendimento che esistono nelle scuole a orario continuato della Svizzera tedesca. L'articolo mette in discussione le aspettative scolastiche nei confronti delle scuole a orario continuato e i modelli di qualità di riferimento in vigore. L'articolo esamina inoltre le motivazioni pedagogiche che prevalgono nella fondazione di queste scuole, la partecipazione dei direttori e degli insegnanti durante la fondazione, i dispositivi di sostegno differenziato proposti e se questi sono influenzati dagli sviluppi attuali in campo educativo.

Parole chiave: Scuole a orario continuato, proposte di promozione, equità scolastica, qualità delle scuole a orario continuato, sviluppo scolastico.

\section{Educational expectations of all-day schools: Educational promotion at open-attendance all-day schools in Switzerland}

\section{Summary}

In current debates on education, all-day schools are granted a central role especially in view of educational equity and improved individual educational support. However, whether all-day schools in the German-speaking part of Switzerland indeed offer learning opportunities in accordance with that is not known. This paper discusses educational expectations placed on all-day schools as well as current quality models and, looking at all-day schools in the Germanspeaking part of Switzerland, examines what role educational motives play in the establishing of all-day schools, to what extent school principals and teachers participate in conceptual development, what individualizing and differentiating elements of educational promotion are anchored within the offerings of all-day schools, and whether the offerings are influenced by features of school development.

Keywords: All-day schools, educational promotion, educational equity, all-day school quality, school development 
Abbildungsverzeichnis

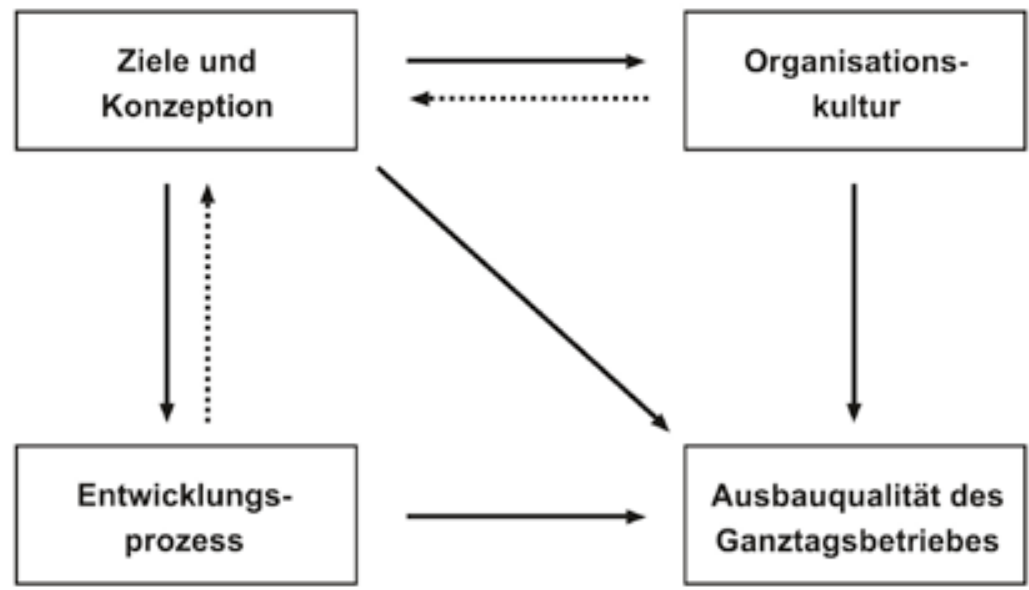

Abbildung 1. Theoretisches Rahmenmodell zur Erklärung der Ausbauqualität des Ganztagsbetriebes (Holtappels \& Rollett, 2009, S. 23).

Cluster $1(n=35)$

Hausaufgabenbetreuung
Hausaufgabenhiffe
Forderangebole tür SuS mit
schwachen Schulteistungen
Förderangebote forr SuS mit
sehr guten Schulieistungen
Förderangebote für
fromdsprachige SuS

ㅁNein ㅁ.Ja, morsatlich

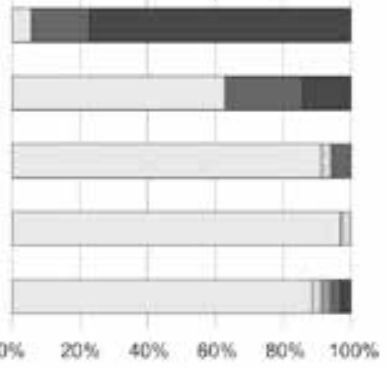

E Ja, mehrmals im Nanat 1 ba, wochentlich
Cluster $2(n=17)$

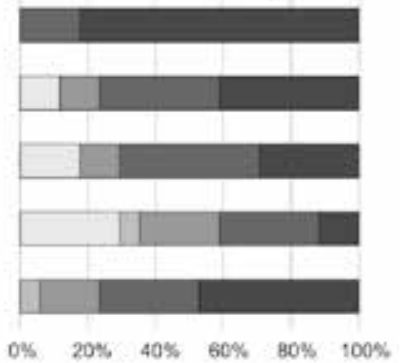

E. mehrmais cie Woche

Abbildung 2. Verfügbarkeit von Förderangeboten in Abhängigkeit der Clusterzugehörigkeit. 


\section{Tabel Lenverzeichnis}

Tabelle 1. Deskriptive Ergebnisse zu den Schulentwicklungsmerkmalen.

\begin{tabular}{lcccc}
\hline & \# Items & $\begin{array}{c}\text { Skalie- } \\
\text { rung }\end{array}$ & M & SD \\
\hline Pädagogische Motive & 4 & $0-3$ & 1.36 & 0.65 \\
Konzeptionelle Festlegungen zur Organisation & 9 & $0-3$ & 1.44 & 0.67 \\
Partizipation durch Schulleitung & 1 & $0-3$ & 1.62 & 1.25 \\
Partizipation durch Lehrpersonen & 1 & $0-3$ & 0.38 & 0.80 \\
\hline
\end{tabular}

Anmerkungen: $N=52$

Tabelle 2. Antworthäufgkeiten zu den pädagogischen Motiven sowie zur konzeptionellen Partizipation.

\begin{tabular}{lcccc}
\hline Pädagogische Motive & $\begin{array}{c}\text { Trifft gar } \\
\text { nicht zu }\end{array}$ & $\begin{array}{c}\text { Trifft eher } \\
\text { nicht zu }\end{array}$ & $\begin{array}{c}\text { Trifft } \\
\text { eher zu }\end{array}$ & $\begin{array}{c}\text { Trifft voll } \\
\text { und ganz } \\
\text { zu }\end{array}$ \\
\hline Sozialer Bedarf in der Gemeinde & $7.69 \%$ & $9.62 \%$ & $34.62 \%$ & $48.08 \%$ \\
Wunsch der unterrichtenden Lehrpersonen & $40.38 \%$ & $34.62 \%$ & $25.00 \%$ & $0.00 \%$ \\
Verbesserung der Bildungschancen & $23.08 \%$ & $28.85 \%$ & $38.46 \%$ & $9.62 \%$ \\
Verbesserung der individuellen Förderung & $26.92 \%$ & $48.08 \%$ & $21.15 \%$ & $3.85 \%$ \\
\hline Konzeptionelle Partizipation & Gar nicht & $\begin{array}{c}\text { In } \\
\text { geringem } \\
\text { Umfang }\end{array}$ & $\begin{array}{c}\text { In } \\
\text { mässigem } \\
\text { Umfang }\end{array}$ & $\begin{array}{c}\text { In hohem } \\
\text { Umfang }\end{array}$ \\
\hline Partizipation durch Schulleitung & $32.69 \%$ & $5.77 \%$ & $28.85 \%$ & $32.69 \%$ \\
Partizipation durch Lehrpersonen & $75.00 \%$ & $17.31 \%$ & $1.92 \%$ & $5.77 \%$ \\
\hline
\end{tabular}

Anmerkungen: $N=52$

Tabelle 3. Antworthäufigkeiten hinsichtlich der Verfügbarkeit von Förderangeboten.

\begin{tabular}{lcccccc}
\hline & $\begin{array}{c}\text { Nicht } \\
\text { vorhanden }\end{array}$ & $\begin{array}{c}\text { Monat- } \\
\text { lich }\end{array}$ & $\begin{array}{c}\text { Mehrmals } \\
\text { im Monat }\end{array}$ & $\begin{array}{c}\text { Wöchent- } \\
\text { lich }\end{array}$ & $\begin{array}{c}\text { Mehrmals } \\
\text { die Woche }\end{array}$ & Täglich \\
\hline Hausaufgabenbetreuung & $3.85 \%$ & $0.00 \%$ & $0.00 \%$ & $0.00 \%$ & $17.31 \%$ & $78.85 \%$ \\
$\begin{array}{l}\text { Hausaufgabenhilfe } \\
\begin{array}{l}\text { Förderangebote für SuS mit } \\
\text { schwachen Schulleistungen }\end{array}\end{array}$ & $67.15 \%$ & $0.00 \%$ & $0.00 \%$ & $3.85 \%$ & $26.92 \%$ & $23.08 \%$ \\
$\begin{array}{l}\text { Förderangebote für SuS mit } \\
\text { sehr guten Schulleistungen }\end{array}$ & $75.00 \%$ & $1.92 \%$ & $0.00 \%$ & $3.85 \%$ & $17.31 \%$ & $9.62 \%$ \\
$\begin{array}{l}\text { Förderangebote für } \\
\text { fremdsprachige SuS }\end{array}$ & $59.62 \%$ & $1.92 \%$ & $1.92 \%$ & $7.69 \%$ & $11.54 \%$ & $17.31 \%$ \\
\hline
\end{tabular}

Anmerkungen: SuS=Schülerinnen und Schüler; $N=52$ 
Tabelle 4. Logistische Regression zur Vorhersage der Clusterzugehörigkeit ( $O=$ Aufgabenbetreunng / $1=$ Aufgabenbetreunng und weitere Förderangebote).

\begin{tabular}{lcccc}
\hline Prädiktoren & $\begin{array}{c}\text { Modell 1 } \\
\operatorname{Exp}(\mathbf{b})\end{array}$ & $\begin{array}{c}\text { Modell 2 } \\
\operatorname{Exp}(\mathbf{b})\end{array}$ & $\begin{array}{c}\text { Modell 3 } \\
\operatorname{Exp}(\mathbf{b})\end{array}$ & $\begin{array}{c}\text { Modell 4 } \\
\operatorname{Exp}(\mathbf{b})\end{array}$ \\
\hline Pädagogische Motive & 1.88 & 1.90 & 1.78 & 1.24 \\
Konzeptionelle Festlegungen & & $3.36^{*}$ & $4.97^{* *}$ & $4.53^{*}$ \\
Partizipation durch LP & & & 3.73 & 2.76 \\
Partizipation durch SL & & & 1.59 & 1.60 \\
Alter der Tagesschule & & & & 1.19 \\
Konstante & $0.20^{*}$ & $0.03^{* *}$ & $0.01^{* *}$ & $0.00^{* *}$ \\
$\mathrm{R}^{2}$ (Nagelkerke) & 0.05 & 0.20 & 0.37 & 0.44 \\
$\mathrm{Chi}^{2}$ & 1.77 & $8.05^{*}$ & $16.09^{* *}$ & $19.57^{* *}$ \\
$\Delta \mathrm{Chi}^{2}$ & 1.77 & $6.28^{*}$ & $8.05^{*}$ & 3.48 \\
\hline
\end{tabular}

Anmerkungen:LP=Lehrpersonen, $S L=$ Schulleitung; ${ }^{*} p \leq .05 ;{ }^{* *} p \leq .01 ; N=52$ 
